\title{
ON CERTAIN WEIGHTED 7-COLORED PARTITIONS
}

\author{
SHANE CHERN AND DAZHAO TANG
}

\begin{abstract}
Inspired by Andrews' 2-colored generalized Frobenius partitions, we consider certain weighted 7-colored partition functions and establish some interesting Ramanujantype identities and congruences. Moreover, we provide combinatorial interpretations of some congruences modulo 5 and 7 . Finally, we study the properties of weighted 7-colored partitions weighted by the parity of certain partition statistics.
\end{abstract}

\section{InTRODUCTION}

In his 1984 Memoir of the American Mathematical Society, Andrews [2] introduced the generalized Frobenius partition or simply the F-partition of $n$, which is a two-rowed array of nonnegative integers

$$
\left(\begin{array}{llll}
a_{1} & a_{2} & \cdots & a_{r} \\
b_{1} & b_{2} & \cdots & b_{r}
\end{array}\right),
$$

wherein each row, which is of the same length, is arranged in non-increasing order with $n=r+\sum_{i=1}^{r} a_{i}+\sum_{i=1}^{r} b_{i}$. Furthermore, Andrews studied many general classes of Fpartitions. One of them is F-partitions whose parts are taken from $k$ copies of the nonnegative integers, which is called $k$-colored $F$-partitions. Let $c \phi_{k}(n)$ denote the number of $k$-colored F-partitions of $n$. Andrews derived the following generating function for $c \phi_{2}(n)$.

Theorem 1.1 (Eq. (5.17), [2]). We have

$$
\sum_{n=0}^{\infty} c \phi_{2}(n) q^{n}=\frac{\left(q^{2} ; q^{4}\right)_{\infty}}{\left(q ; q^{2}\right)_{\infty}^{4}\left(q^{4} ; q^{4}\right)_{\infty}} .
$$

Here and in the sequel, we adopt the following customary notations on partitions and $q$-series:

$$
\begin{aligned}
(a ; q)_{\infty} & :=\prod_{n=0}^{\infty}\left(1-a q^{n}\right), \\
\left(a_{1}, a_{2}, \cdots, a_{n} ; q\right)_{\infty} & :=\left(a_{1} ; q\right)_{\infty}\left(a_{2} ; q\right)_{\infty} \cdots\left(a_{n} ; q\right)_{\infty}, \quad|q|<1 .
\end{aligned}
$$

In addition, Andrews obtained the following congruence modulo 5 for $c \phi_{2}(n)$.

Date: October 14, 2018.

2010 Mathematics Subject Classification. 05A17, 11P83, 05A30.

Key words and phrases. Weighted 7-colored partition; Ramanujan-type congruence; Unified multirank; Vector crank. 
Theorem 1.2 (Corollary 10.1, [2]). For all $n \geq 0$,

$$
c \phi_{2}(5 n+3) \equiv 0 \quad(\bmod 5) .
$$

Moreover, Andrews defined the D-rank of an F-partition (1.1) to be $a_{1}-b_{1}$ (if the parts are colored, the D-rank is the numerical magnitude of $\left.a_{1}-b_{1}\right)$. He then conjectured [2, Conjecture 11.1] that the D-rank might explain the congruence (1.3) combinatorially for 2-colored F-partitions. Unfortunately, this was asserted untrue by Lovejoy [17].

According to the generating function (1.2) for $c \phi_{2}(n)$ and the second author's recent work with Shishuo $\mathrm{Fu} \mathrm{[10]} \mathrm{involving} \mathrm{classical} \mathrm{theta} \mathrm{functions,} \mathrm{in} \mathrm{this} \mathrm{paper,} \mathrm{we} \mathrm{study} \mathrm{the} \mathrm{following}$ weighted 7-colored partitions $w_{t}(n)$ arithmetically as well as combinatorially, given by

$$
\sum_{n=0}^{\infty} w_{t}(n) q^{n}:=\frac{\left(q^{2} ; q^{2}\right)_{\infty}}{\left(q ; q^{2}\right)_{\infty}^{4}\left(q^{t} ; q^{t}\right)_{\infty}^{2}} .
$$

In some sense, this partition function can be viewed as a generalization of Andrews' $c \phi_{2}(n)$, as one may notice that $w_{4}(n)=c \phi_{2}(n)$ for all $n \geq 0$.

Similar to $c \phi_{2}(n)$, there are some Ramanujan-type congruences for $w_{t}(n)$. Below are several examples that we will prove in the later sections. When $t \equiv 0(\bmod 3)$, we have

$$
\begin{aligned}
& w_{t}(3 n+1) \equiv 0 \quad(\bmod 4), \\
& w_{t}(3 n+2) \equiv 0 \quad(\bmod 9) .
\end{aligned}
$$

Furthermore, we have

$$
\begin{aligned}
& w_{t}(5 n+3) \equiv w_{t}(5 n+4) \equiv 0 \quad(\bmod 5), \quad \text { if } t \equiv 0 \quad(\bmod 5), \\
& w_{t}(5 n+4) \equiv 0 \quad(\bmod 5), \quad \text { if } t \equiv 1 \quad(\bmod 5), \\
& w_{t}(5 n+3) \equiv 0 \quad(\bmod 5), \quad \text { if } t \equiv 4 \quad(\bmod 5) .
\end{aligned}
$$

In addition, we get

$$
\begin{aligned}
w_{2}(7 n+4) \equiv 0 & (\bmod 7), \\
w_{2}(11 n+10) \equiv 0 & (\bmod 11), \\
w_{3}(24 n+23) & \equiv 0 \quad(\bmod 27) .
\end{aligned}
$$

Of course, there are more congruences beyond this list. However, these congruences can be proved by the standard $q$-series techniques and hence we do not require complicated tools like modular forms.

The rest of this paper is organized as follows. In Sect. 2, we establish some Ramanujantype identities and congruences for $w_{t}(n)$. We will then consider a unified multirank and a vector crank which can give combinatorial interpretations of certain congruences modulo 5 and 7 for $w_{t}(n)$ in Sect. 3. In Sect. 4, we study the properties of weighted 7-colored partitions weighted by the parity of certain partition statistics. Some Ramanujan-type congruences and an analog of Euler's recurrence relation are obtained. In the last section, we conclude with some remarks and questions for further study. 


\section{RAMANUJAN-TYPE IDENTITIES AND CONGRUENCES}

In this section, we shall prove some Ramanujan-type identities and congruences for weighted 7-colored partitions $w_{t}(n)$.

For notational convenience, we denote $\left(q^{k} ; q^{k}\right)_{\infty}$ by $f_{k}$ for positive integers $k$ when manipulating $q$-series. Recall that Ramanujan's classical theta functions $\varphi(q)$ and $\psi(q)$ are given by

$$
\begin{aligned}
& \varphi(q):=\sum_{n=-\infty}^{\infty} q^{n^{2}}=\frac{f_{2}^{5}}{f_{1}^{2} f_{4}^{2}}, \\
& \psi(q):=\sum_{n=0}^{\infty} q^{n(n+1) / 2}=\frac{f_{2}^{2}}{f_{1}} .
\end{aligned}
$$

It is also known that

$$
\varphi(-q)=\frac{f_{1}^{2}}{f_{2}} .
$$

Before stating our results, we require the following 2-dissections.

$$
\begin{aligned}
f_{1}^{2} & =\frac{f_{2} f_{8}^{5}}{f_{4}^{2} f_{16}^{2}}-2 q \frac{f_{2} f_{16}^{2}}{f_{8}}, \\
f_{1}^{4} & =\frac{f_{4}^{10}}{f_{2}^{2} f_{8}^{4}}-4 q \frac{f_{2}^{2} f_{8}^{4}}{f_{4}^{2}}, \\
\frac{1}{f_{1}^{4}} & =\frac{f_{4}^{14}}{f_{2}^{14} f_{8}^{4}}+4 q \frac{f_{4}^{2} f_{8}^{4}}{f_{2}^{10}} .
\end{aligned}
$$

These follow respectively from the 2-dissections of $\varphi(-q), \varphi^{2}(-q)$, and $\varphi^{2}(q)$ (cf. [6, p. 40, Entry 25]).

We also need the following 3-dissections of $\psi(q)$ and $1 / \varphi(-q)$,

$$
\begin{aligned}
\psi(q) & =\psi\left(q^{9}\right)\left(\frac{1}{x\left(q^{3}\right)}+q\right), \\
\frac{1}{\varphi(-q)} & =\frac{\varphi\left(-q^{9}\right)^{3}}{\varphi\left(-q^{3}\right)^{4}}\left(1+2 q x\left(q^{3}\right)+4 q^{2} x\left(q^{3}\right)^{2}\right),
\end{aligned}
$$

where

$$
x(q)=\frac{\left(q ; q^{2}\right)_{\infty}}{\left(q^{3} ; q^{6}\right)_{\infty}^{3}} .
$$

Here (2.7) comes directly from the Jacobi's triple product identity. For (2.8), see [4].

Theorem 2.1. For $t \equiv 0(\bmod 2)$,

$$
\sum_{n=0}^{\infty} w_{t}(2 n) q^{n}=\frac{f_{2}^{14}}{f_{1}^{9} f_{4}^{4} f_{t / 2}^{2}}
$$




$$
\sum_{n=0}^{\infty} w_{t}(2 n+1) q^{n}=\frac{4 f_{2}^{2} f_{4}^{4}}{f_{1}^{5} f_{t / 2}^{2}}
$$

Proof. From (2.6) and (1.4), we have

$$
\sum_{n=0}^{\infty} w_{t}(n) q^{n}=\frac{f_{2}^{5}}{f_{1}^{4} f_{t}^{2}}=\frac{f_{4}^{14}}{f_{2}^{9} f_{8}^{4} f_{t}^{2}}+4 q \frac{f_{4}^{2} f_{8}^{4}}{f_{2}^{5} f_{t}^{2}} .
$$

Extracting terms involving $q^{2 n}$ and $q^{2 n+1}$ in (2.12) and replacing $q^{2}$ by $q$, one easily obtains (2.10)-(2.11). This completes the proof.

This immediately yields

Corollary 2.2. For $t \equiv 0(\bmod 2)$,

$$
w_{t}(2 n+1) \equiv 0 \quad(\bmod 4) .
$$

Remark 2.3. We notice that the congruence $w_{4}(2 n+1)=c \phi_{2}(2 n+1) \equiv 0(\bmod 4)$ was first proved by Andrews [2]. Following the same line of proving Theorem 2.1, one may also obtain the 2-dissection of the generating function of $w_{1}(n)$.

Theorem 2.4. For $t \equiv 0(\bmod 3)$,

$$
\begin{aligned}
\sum_{n=0}^{\infty} w_{t}(3 n) q^{n} & =\frac{f_{2}^{4} f_{3}^{4} f_{6}}{f_{1}^{8} f_{t / 3}^{2}}\left(\frac{f_{2}^{2} f_{3}^{6}}{f_{1}^{2} f_{6}^{6}}+10 q \frac{f_{1} f_{6}^{3}}{f_{2} f_{3}^{3}}\right), \\
\sum_{n=0}^{\infty} w_{t}(3 n+1) q^{n} & =4 \frac{f_{2}^{4} f_{3}^{4} f_{6}}{f_{1}^{8} f_{t / 3}^{2}}\left(\frac{f_{2} f_{3}^{3}}{f_{1} f_{6}^{3}}+q \frac{f_{1}^{2} f_{6}^{6}}{f_{2}^{2} f_{3}^{6}}\right), \\
\sum_{n=0}^{\infty} w_{t}(3 n+2) q^{n} & =9 \frac{f_{2}^{4} f_{3}^{4} f_{6}}{f_{1}^{8} f_{t / 3}^{2}}
\end{aligned}
$$

As a consequence of Theorem 2.4, we have

Corollary 2.5. For $t \equiv 0(\bmod 3)$,

$$
\begin{array}{ll}
w_{t}(3 n+1) \equiv 0 & (\bmod 4), \\
w_{t}(3 n+2) \equiv 0 & (\bmod 9) .
\end{array}
$$

To prove Theorem 2.4, we need to show the following lemma.

Lemma 2.6. We have

$$
\frac{f_{2}^{5}}{f_{1}^{4}}=\frac{f_{6}^{4} f_{9}^{4} f_{18}}{f_{3}^{8}}\left(\frac{1}{x\left(q^{3}\right)^{2}}+\frac{4 q}{x\left(q^{3}\right)}+9 q^{2}+10 q^{3} x\left(q^{3}\right)+4 q^{4} x\left(q^{3}\right)^{2}\right) .
$$

Proof. By Eqs. (2.7) and (2.8), we have

$$
\frac{f_{2}^{5}}{f_{1}^{4}}=\frac{\psi(q)^{2}}{\varphi(-q)}
$$




$$
\begin{aligned}
& =\psi\left(q^{9}\right)^{2}\left(\frac{1}{x\left(q^{3}\right)}+q\right)^{2} \frac{\varphi\left(-q^{9}\right)^{3}}{\varphi\left(-q^{3}\right)^{4}}\left(1+2 q x\left(q^{3}\right)+4 q^{2} x\left(q^{3}\right)^{2}\right) \\
& =\frac{f_{6}^{4} f_{9}^{4} f_{18}}{f_{3}^{8}}\left(\frac{1}{x\left(q^{3}\right)^{2}}+\frac{4 q}{x\left(q^{3}\right)}+9 q^{2}+10 q^{3} x\left(q^{3}\right)+4 q^{4} x\left(q^{3}\right)^{2}\right) .
\end{aligned}
$$

This establishes (2.16).

By Lemma 2.6 and Eq. (2.9), we have the following

Corollary 2.7. Let

$$
\sum_{n=0}^{\infty} a(n) q^{n}=\frac{f_{2}^{5}}{f_{1}^{4}}
$$

Then

$$
\begin{aligned}
\sum_{n=0}^{\infty} a(3 n) q^{n} & =\frac{f_{2}^{4} f_{3}^{4} f_{6}}{f_{1}^{8}}\left(\frac{f_{2}^{2} f_{3}^{6}}{f_{1}^{2} f_{6}^{6}}+10 q \frac{f_{1} f_{6}^{3}}{f_{2} f_{3}^{3}}\right), \\
\sum_{n=0}^{\infty} a(3 n+1) q^{n} & =4 \frac{f_{2}^{4} f_{3}^{4} f_{6}}{f_{1}^{8}}\left(\frac{f_{2} f_{3}^{3}}{f_{1} f_{6}^{3}}+q \frac{f_{1}^{2} f_{6}^{6}}{f_{2}^{2} f_{3}^{6}}\right), \\
\sum_{n=0}^{\infty} a(3 n+2) q^{n} & =9 \frac{f_{2}^{4} f_{3}^{4} f_{6}}{f_{1}^{8}}
\end{aligned}
$$

and hence

$$
\begin{aligned}
& a(3 n+1) \equiv 0 \quad(\bmod 4), \\
& a(3 n+2) \equiv 0 \quad(\bmod 9) .
\end{aligned}
$$

Proof of Theorem 2.4. We notice that

$$
\sum_{n=0}^{\infty} w_{t}(n) q^{n}=\frac{f_{2}^{5}}{f_{1}^{4} f_{t}^{2}} .
$$

Since $t \equiv 0(\bmod 3)$, Theorem 2.4 is a direct consequence of Corollary 2.7.

With the help of Eq. (2.15), we also have

Theorem 2.8. For all $n \geq 0$,

$$
w_{3}(24 n+23) \equiv 0 \quad(\bmod 27) .
$$

Proof. Notice that

$$
\sum_{n=0}^{\infty} w_{t}(3 n+2) q^{n}=9 \frac{f_{2}^{4} f_{3}^{4} f_{6}}{f_{1}^{8} f_{t / 3}^{2}} .
$$

Hence to prove (2.17), it suffices to show

$$
a_{1}(8 n+7) \equiv 0 \quad(\bmod 3)
$$


where

$$
\sum_{n=0}^{\infty} a_{1}(n) q^{n}=\frac{f_{2}^{4} f_{3}^{4} f_{6}}{f_{1}^{10}}
$$

With the help of (2.4), it follows that, modulo 3,

$$
\begin{aligned}
\sum_{n=0}^{\infty} a_{1}(n) q^{n} & =\frac{f_{2}^{4} f_{3}^{4} f_{6}}{f_{1}^{10}} \equiv f_{1}^{2} f_{2}^{7}=\left(\frac{f_{2} f_{8}^{5}}{f_{4}^{2} f_{16}^{2}}-2 q \frac{f_{2} f_{16}^{2}}{f_{8}}\right) f_{2}^{7} \\
& =\frac{f_{2}^{8} f_{8}^{5}}{f_{4}^{2} f_{16}^{2}}-2 q \frac{f_{2}^{8} f_{16}^{2}}{f_{8}} .
\end{aligned}
$$

We now extract terms involving $q^{2 n+1}$ and replace $q^{2}$ by $q$, then

$$
\sum_{n=0}^{\infty} a_{1}(2 n+1) q^{n} \equiv-2 \frac{f_{1}^{8} f_{8}^{2}}{f_{4}} .
$$

Through a similar argument, we have

$$
\sum_{n=0}^{\infty} a_{1}(4 n+3) q^{n} \equiv 16 f_{2}^{7} f_{4}^{2}
$$

which contains no terms of the form $q^{2 n+1}$. Hence $a_{1}(4(2 n+1)+3)=a_{1}(8 n+7) \equiv 0$ $(\bmod 3)$.

Remark 2.9. As pointed out by the referee, (2.17) still holds modulo $3^{6}=729$, which can be proved via modular forms. However, it is unclear whether there is an elementary proof.

We also have some congruences modulo 5 .

Theorem 2.10. For all $n \geq 0$,

$$
\begin{aligned}
& w_{t}(5 n+3) \equiv w_{t}(5 n+4) \equiv 0 \quad(\bmod 5), \quad \text { if } t \equiv 0 \quad(\bmod 5), \\
& w_{t}(5 n+4) \equiv 0 \quad(\bmod 5), \quad \text { if } t \equiv 1 \quad(\bmod 5), \\
& w_{t}(5 n+3) \equiv 0 \quad(\bmod 5), \quad \text { if } t \equiv 4 \quad(\bmod 5)
\end{aligned}
$$

Proof. If $t \equiv 0(\bmod 5)$, we have

$$
\sum_{n=0}^{\infty} w_{t}(n) q^{n} \equiv(q ; q)_{\infty} \frac{\left(q^{10} ; q^{10}\right)_{\infty}}{\left(q^{5} ; q^{5}\right)_{\infty}\left(q^{t} ; q^{t}\right)_{\infty}^{2}} \quad(\bmod 5)
$$

From Euler's pentagonal number theorem [1, p. 11, Corollary 1.7], which tells

$$
(q ; q)_{\infty}=\sum_{n=-\infty}^{\infty}(-1)^{n} q^{n(3 n+1) / 2},
$$

we notice that $(q ; q)_{\infty}$ has no terms in which the power of $q$ is 3 or $4 \bmod 5$. Hence

$$
w_{t}(5 n+3) \equiv 0 \quad(\bmod 5) \text {, }
$$


and

We next notice that

$$
w_{t}(5 n+4) \equiv 0 \quad(\bmod 5)
$$

$$
\sum_{n=0}^{\infty} w_{t}(n) q^{n} \equiv(q ; q)_{\infty}\left(q^{t} ; q^{t}\right)_{\infty}^{3} \frac{\left(q^{10} ; q^{10}\right)_{\infty}}{\left(q^{5} ; q^{5}\right)_{\infty}\left(q^{5 t} ; q^{5 t}\right)_{\infty}} \quad(\bmod 5) .
$$

Recall that the Jacobi's identity [7, p. 14, Theorem 1.3.9] tells

$$
(q ; q)_{\infty}^{3}=\sum_{n=0}^{\infty}(-1)^{n}(2 n+1) q^{n(n+1) / 2} .
$$

From (2.21) and (2.22), one readily has

$$
(q ; q)_{\infty}=E_{0}+E_{1}+E_{2},
$$

where $E_{i}$ consists of those terms in which the power of $q$ is $i$ modulo 5 , and

$$
(q ; q)_{\infty}^{3}=J_{0}+J_{1}+J_{3},
$$

where $J_{i}$ consists of those terms in which the power of $q$ is $i$ modulo 5 . Furthermore, we note that $J_{3} \equiv 0(\bmod 5)$, so

$$
(q ; q)_{\infty}^{3} \equiv J_{0}+J_{1} \quad(\bmod 5) .
$$

If $t \equiv 1(\bmod 5)$, we observe that

$$
\left(q^{t} ; q^{t}\right)_{\infty}^{3} \equiv J_{0}^{*}+J_{1}^{*} \quad(\bmod 5)
$$

where $J_{i}^{*}$ consists of terms in which the power of $q$ is $i$ modulo 5 . It follows that,

$$
(q ; q)_{\infty}\left(q^{t} ; q^{t}\right)_{\infty}^{3} \equiv\left(E_{0}+E_{1}+E_{2}\right)\left(J_{0}^{*}+J_{1}^{*}\right) \quad(\bmod 5)
$$

which contains no terms of the form $q^{5 n+4}$. Hence, $w_{t}(5 n+4) \equiv 0(\bmod 5)$.

If $t \equiv 4(\bmod 5)$, we have

$$
\left(q^{t} ; q^{t}\right)_{\infty}^{3} \equiv J_{0}^{*}+J_{4}^{*} \quad(\bmod 5)
$$

The rest of the proof is similar.

For $w_{2}(n)$, we have the following $\bmod 7$ congruence.

Theorem 2.11. For all $n \geq 0$,

$$
w_{2}(7 n+4) \equiv 0 \quad(\bmod 7) .
$$

Proof. Notice that

$$
\sum_{n=0}^{\infty} w_{2}(n) q^{n}=\frac{\left(q^{2} ; q^{2}\right)_{\infty}}{\left(q ; q^{2}\right)_{\infty}^{4}\left(q^{2} ; q^{2}\right)_{\infty}^{2}}=\frac{\left(q^{2} ; q^{2}\right)_{\infty}^{3}}{(q ; q)_{\infty}^{4}} \equiv \frac{(q ; q)_{\infty}^{3}\left(q^{2} ; q^{2}\right)_{\infty}^{3}}{\left(q^{7} ; q^{7}\right)_{\infty}} \quad(\bmod 7)
$$

Similarly, (2.22) tells

and hence

$$
(q ; q)_{\infty}^{3} \equiv J_{0}+J_{1}+J_{3} \quad(\bmod 7)
$$

$$
\left(q^{2} ; q^{2}\right)_{\infty}^{3} \equiv J_{0}^{*}+J_{2}^{*}+J_{6}^{*} \quad(\bmod 7)
$$


This time $J_{i}$ and $J_{i}^{*}$ consist of terms in which the power of $q$ is $i$ modulo 7 .

It follows that, modulo 7 ,

$$
(q ; q)_{\infty}^{3}\left(q^{2} ; q^{2}\right)_{\infty}^{3} \equiv\left(J_{0}+J_{1}+J_{3}\right)\left(J_{0}^{*}+J_{2}^{*}+J_{6}^{*}\right),
$$

which contains no terms of the form $q^{7 n+4}$. Hence, $w_{2}(7 n+4) \equiv 0(\bmod 7)$.

Finally, we have a mod 11 congruence for $w_{2}(n)$.

Theorem 2.12. For all $n \geq 0$,

$$
w_{2}(11 n+10) \equiv 0 \quad(\bmod 11) .
$$

Proof. It is easy to see that

$$
\sum_{n=0}^{\infty} w_{2}(n) q^{n}=\frac{\left(q^{2} ; q^{2}\right)_{\infty}^{3}}{(q ; q)_{\infty}^{4}} \equiv \frac{1}{\left(q^{22} ; q^{22}\right)_{\infty}} \frac{\left(q^{2} ; q^{2}\right)_{\infty}^{14}}{(q ; q)_{\infty}^{4}} \quad(\bmod 11)
$$

Hence it suffices to prove that

$$
a_{2}(11 n+10) \equiv 0 \quad(\bmod 11),
$$

where

$$
\sum_{n=0}^{\infty} a_{2}(n) q^{n}=\frac{\left(q^{2} ; q^{2}\right)_{\infty}^{14}}{(q ; q)_{\infty}^{4}}
$$

According to Theorem 2 in [9], let $p=11$ and $(r, s)=(-4,14)$, we have

$$
a_{2}(11 n+120)=11^{4} a_{2}\left(\frac{n}{11}\right),
$$

where $a_{2}(\alpha)=0$ is $\alpha$ is not a nonnegative integer. This along with the verification of the first several $n$ 's finish our proof.

\section{Combinatorial interpretations}

3.1. A unified multirank. In this section, we give combinatorial interpretations of Theorems 2.10 and 2.11. In doing so, we introduce a multirank and a vector crank for weighted 7-colored partitions. This multirank (resp. vector crank) function enables us to divide its corresponding partition set into five (resp. seven) equivalence classes.

For a given partition $\lambda$, we let $\ell(\lambda)$ denote the number of parts in $\lambda$ and $\sigma(\lambda)$ denote the sum of all parts in $\lambda$ with the convention $\ell(\lambda)=\sigma(\lambda)=0$ for empty partition $\lambda$ of 0 . Let $\mathcal{P}$ denote the set of all ordinary partitions, and $\mathcal{O}$ (resp. $\mathcal{D E}, \mathcal{D O}$ ) denote the set of all partitions into odd parts (resp. distinct even parts, distinct odd parts).

Let

$$
\mathcal{V}_{t}=\left\{\left(\lambda_{1}, \lambda_{2}, \lambda_{3}, \lambda_{4}, \lambda_{5}, t \lambda_{6}, t \lambda_{7}\right) \mid \lambda_{1} \in \mathcal{D} \mathcal{E}, \lambda_{2}, \lambda_{3}, \lambda_{4}, \lambda_{5} \in \mathcal{O}, \lambda_{6}, \lambda_{7} \in \mathcal{P}\right\}
$$

For a vector (weighted 7-colored) partition $\vec{\lambda} \in \mathcal{V}_{t}$, we define the sum of parts function $s$, the weight function $w t$, and the multirank function $r_{7}$ by

$$
s(\vec{\lambda})=\sigma\left(\lambda_{1}\right)+\sigma\left(\lambda_{2}\right)+\sigma\left(\lambda_{3}\right)+\sigma\left(\lambda_{4}\right)+\sigma\left(\lambda_{5}\right)+t \sigma\left(\lambda_{6}\right)+t \sigma\left(\lambda_{7}\right),
$$




$$
\begin{aligned}
& w t(\vec{\lambda})=(-1)^{\ell\left(\lambda_{1}\right)}, \\
& r_{7}(\vec{\lambda})=\ell\left(\lambda_{2}\right)-\ell\left(\lambda_{3}\right)+2\left(\ell\left(\lambda_{4}\right)-\ell\left(\lambda_{5}\right)\right)+2\left(\ell\left(\lambda_{6}\right)-\ell\left(\lambda_{7}\right)\right) .
\end{aligned}
$$

Remark 3.1. We note that for the purpose of combinatorially obtaining Ramanujan-type congruences, there are a number of alternatives that will work equally well as multirank $r_{7}$. For example, we can use $\ell\left(\lambda_{2}\right)-\ell\left(\lambda_{3}\right)+2\left(\ell\left(\lambda_{4}\right)-\ell\left(\lambda_{5}\right)\right)+h\left(\ell\left(\lambda_{6}\right)-\ell\left(\lambda_{7}\right)\right)$ for $h \not \equiv 0$ $(\bmod 5)$ to replace the right hand side of $r_{7}$ in $(3.3)$.

The weighted count of vector partitions of $n$ with multirank equal to $m$, denoted by $N_{\mathcal{V}_{t}}(m, n)$, is given by

$$
N_{\mathcal{V}_{t}}(m, n)=\sum_{\substack{\vec{\lambda} \in \mathcal{V}_{t, s}(\vec{\lambda})=n \\ r_{7}(\vec{\lambda})=m}} w t(\vec{\lambda})
$$

We also define the weighted count of vector partitions of $n$ with multirank congruent to $k$ modulo $m$ by

$$
N_{\mathcal{V}_{t}}(k, m, n)=\sum_{j=-\infty}^{\infty} N_{\mathcal{V}_{t}}(j m+k, n)=\sum_{\substack{\vec{\lambda} \in \mathcal{V}_{t}, s(\vec{\lambda})=n \\ r_{7}(\vec{\lambda}) \equiv k \\(\bmod m)}} w t(\vec{\lambda}) .
$$

Thus we have the following generating function for $N_{\mathcal{V}_{t}}(m, n)$ :

$$
\sum_{m=-\infty}^{\infty} \sum_{n=0}^{\infty} N_{\mathcal{V}_{t}}(m, n) z^{m} q^{n}=\frac{\left(q^{2} ; q^{2}\right)_{\infty}}{\left(z q, z^{-1} q, z^{2} q, z^{-2} q ; q^{2}\right)_{\infty}\left(z^{2} q^{t}, z^{-2} q^{t} ; q^{t}\right)_{\infty}}
$$

According to the definition of $N_{\mathcal{V}_{t}}(m, n)$, it is nontrivial that $N_{\mathcal{V}_{t}}(m, n)$ is always nonnegative. However, the following corollary of the $q$-binomial theorem proved by Berkovich and Garvan [5] gives us an affirmative answer.

Proposition 3.2. If $|q|,|z|<1$, then

$$
\frac{(a z ; q)_{\infty}}{(a ; q)_{\infty}(z ; q)_{\infty}}=\frac{1}{(a ; q)_{\infty}}+\sum_{n=1}^{\infty} \frac{z^{n}}{\left(a q^{n} ; q\right)_{\infty}(q ; q)_{n}} .
$$

This immediately yields the following corollary.

Corollary 3.3. The coefficients $N_{\mathcal{V}_{t}}(m, n)$ defined in (3.4) are always nonnegative.

Theorem 3.4. The following relations hold for all $n \geq 0, j \equiv 1(\bmod 5), k \equiv 4(\bmod 5)$ and $l \equiv 0(\bmod 5)$.

$$
\begin{aligned}
& N_{\mathcal{V}_{j}}(0,5,5 n+4)=N_{\mathcal{V}_{j}}(1,5,5 n+4)=\cdots=N_{\mathcal{V}_{j}}(4,5,5 n+4)=\frac{w_{j}(5 n+4)}{5} \\
& N_{\mathcal{V}_{k}}(0,5,5 n+3)=N_{\mathcal{V}_{k}}(1,5,5 n+3)=\cdots=N_{\mathcal{V}_{k}}(4,5,5 n+3)=\frac{w_{k}(5 n+3)}{5} \\
& N_{\mathcal{V}_{l}}(0,5,5 n+3)=N_{\mathcal{V}_{l}}(1,5,5 n+3)=\cdots=N_{\mathcal{V}_{l}}(4,5,5 n+3)=\frac{w_{l}(5 n+3)}{5}
\end{aligned}
$$




$$
N_{\mathcal{V}_{l}}(0,5,5 n+4)=N_{\mathcal{V}_{l}}(1,5,5 n+4)=\cdots=N_{\mathcal{V}_{l}}(4,5,5 n+4)=\frac{w_{l}(5 n+4)}{5}
$$

To prove Theorem 3.4, the main ingredient is the following modified Jacobi's triple product identity [12]:

$$
\prod_{n=1}^{\infty}\left(1-q^{n}\right)\left(1-z q^{n}\right)\left(1-z^{-1} q^{n}\right)=\sum_{n=0}^{\infty}(-1)^{n} q^{n(n+1) / 2} z^{-n}\left(\frac{1-z^{2 n+1}}{1-z}\right) .
$$

Proof of Theorem 3.4. We will present the proof of the case $j \equiv 1(\bmod 5)$ to illustrate the main idea. The proofs of the remaining cases are similar.

Putting $z=\zeta_{5}=e^{2 \pi i / 5}$ and $t=j=5 s+1$ in (3.4), we see that

$$
\begin{aligned}
& \sum_{m=-\infty}^{\infty} \sum_{n=0}^{\infty} N_{\mathcal{V}_{j}}(m, n) \zeta_{5}^{m} q^{n} \\
& =\sum_{n=0}^{\infty} \sum_{i=0}^{4} N_{\mathcal{V}_{j}}(i, 5, n) \zeta_{5}^{i} q^{n} \\
& =\frac{\left(q^{2} ; q^{2}\right)_{\infty}}{\left(\zeta_{5} q, \zeta_{5}^{-1} q, \zeta_{5}^{2} q, \zeta_{5}^{-2} q ; q^{2}\right)_{\infty}\left(\zeta_{5}^{2} q^{5 s+1}, \zeta_{5}^{-2} q^{5 s+1} ; q^{5 s+1}\right)_{\infty}} \\
& =\frac{(q ; q)_{\infty}\left(\zeta_{5} q^{5 s+1}, \zeta_{5}^{-1} q^{5 s+1}, q^{5 s+1} ; q^{5 s+1}\right)_{\infty}}{\left(q^{5} ; q^{10}\right)_{\infty}\left(q^{5(5 s+1)} ; q^{5(5 s+1)}\right)_{\infty}} \\
& =\frac{\sum_{m=-\infty}^{\infty} \sum_{n=0}^{\infty}(-1)^{m+n} q^{m(3 m-1) / 2+n(n+1)(5 s+1) / 2} \zeta_{5}^{-n}\left(1-\zeta_{5}^{2 n+1}\right)}{\left(q^{5} ; q^{10}\right)_{\infty}\left(q^{5(5 s+1)} ; q^{5(5 s+1)}\right)_{\infty}\left(1-\zeta_{5}\right)}
\end{aligned}
$$

Here the last equality relies on $(2.21)$ and (3.9). Since $m(3 m-1) / 2 \equiv 0,1,2(\bmod 5)$ and $n(n+1)(5 s+1) / 2 \equiv 0,1,3(\bmod 5)$, it follows that $m(3 m-1) / 2+n(n+1)(5 s+1) / 2$ is congruent to 4 modulo 5 exactly when $m \equiv 1(\bmod 5)$ and $n \equiv 2(\bmod 5)$. This means that the coefficient of $q^{5 n+4}$ in

$$
(-1)^{m+n} q^{m(3 m-1) / 2+n(n+1)(5 s+1) / 2} \zeta_{5}^{-n}\left(1-\zeta_{5}^{2 n+1}\right)
$$

is zero since $\zeta_{5}^{-n}\left(1-\zeta_{5}^{2 n+1}\right)=0$ when $n \equiv 2(\bmod 5)$. Thus

$$
\sum_{i=0}^{4} N_{\mathcal{V}_{j}}(i, 5,5 n+4) \zeta_{5}^{i}=0
$$

We note that the left hand side of (3.10) is a polynomial in $\zeta_{5}$ over $\mathbb{Z}$. It follows that $N_{\mathcal{V}_{j}}(i, 5,5 n+4)$ has the same value for all $0 \leq i \leq 4$, since the minimal polynomial for $\zeta_{5}$ over $\mathbb{Q}$ is

$$
1+\zeta_{5}+\zeta_{5}^{2}+\zeta_{5}^{3}+\zeta_{5}^{4}
$$

We therefore establish $(3.5)$ for $j \equiv 1(\bmod 5)$. This ends our proof. 
TABLE 1. Multiank for 7-colored partitions $w_{4}(3)$

\begin{tabular}{|c|c||c|c|}
$\vec{\pi}$ & $\left(w t(\vec{\pi}), r_{7}(\vec{\pi})\right)$ & $\vec{\pi}$ & $\left(w t(\vec{\pi}), r_{7}(\vec{\pi})\right)$ \\
$3_{2}$ & $(1,1)$ & $1_{2}+1_{2}+1_{5}$ & $(1,0)$ \\
$3_{3}$ & $(1,-1)$ & $1_{2}+1_{3}+1_{3}$ & $(1,-1)$ \\
$3_{4}$ & $(1,2)$ & $1_{3}+1_{3}+1_{4}$ & $(1,0)$ \\
$3_{5}$ & $(1,-2)$ & $1_{3}+1_{3}+1_{5}$ & $(1,-4)$ \\
$2_{1}+1_{2}$ & $(-1,1)$ & $1_{2}+1_{4}+1_{4}$ & $(1,5)$ \\
$2_{1}+1_{3}$ & $(-1,-1)$ & $1_{3}+1_{4}+1_{4}$ & $(1,3)$ \\
$2_{1}+1_{4}$ & $(-1,2)$ & $1_{4}+1_{4}+1_{5}$ & $(1,2)$ \\
$2_{1}+1_{5}$ & $(-1,-2)$ & $1_{2}+1_{5}+1_{5}$ & $(1,-3)$ \\
$1_{2}+1_{2}+1_{2}$ & $(1,3)$ & $1_{3}+1_{5}+1_{5}$ & $(1,-5)$ \\
$1_{3}+1_{3}+1_{3}$ & $(1,-3)$ & $1_{4}+1_{5}+1_{5}$ & $(1,-2)$ \\
$1_{4}+1_{4}+1_{4}$ & $(1,6)$ & $1_{2}+1_{3}+1_{4}$ & $(1,2)$ \\
$1_{5}+1_{5}+1_{5}$ & $(1,-6)$ & $1_{2}+1_{3}+1_{5}$ & $(1,-2)$ \\
$1_{2}+1_{2}+1_{3}$ & $(1,1)$ & $1_{2}+1_{4}+1_{5}$ & $(1,1)$ \\
$1_{2}+1_{2}+1_{4}$ & $(1,4)$ & $1_{3}+1_{4}+1_{5}$ & $(1,-1)$
\end{tabular}

Example 3.5. In Table 1, we list the multirank $r_{7}$ for the total 28 weighted 7 -colored partitions of 3 . Note that the weighted count of multirank divides $w_{4}(3)=20$ into five residue classes, each with equal size 4 .

3.2. A vector crank. For a given partition $\lambda$, the crank $c(\lambda)$ of $\lambda$ is given by [3]:

$$
c(\lambda):= \begin{cases}\lambda_{1}, & \text { if } n_{1}(\lambda)=0 \\ \mu(\lambda)-n_{1}(\lambda), & \text { if } n_{1}(\lambda)>0,\end{cases}
$$

where $n_{1}(\lambda)$ is the number of 1's in $\lambda, \lambda_{1}$ is the largest part in $\lambda$, and $\mu(\lambda)$ is the number of parts larger than $n_{1}(\lambda)$. By extending the partition set $\mathcal{P}$ to a new set $\mathcal{P}^{*}$, in which two additional copies of the partition 1 (say $1^{*}$ and $1^{* *}$ ) are added, B. Kim [14,15] obtained

$$
\frac{(q ; q)_{\infty}}{\left(z q, z^{-1} q ; q\right)_{\infty}}=\sum_{\lambda \in \mathcal{P}} w t^{*}(\lambda) z^{c^{*}(\lambda)} q^{\sigma^{*}(\lambda)}
$$

where $w t^{*}(\lambda), c^{*}(\lambda)$ and $\sigma^{*}(\lambda)$ are defined as follows:

$$
\begin{aligned}
& w t^{*}(\lambda):= \begin{cases}1, & \text { if } \lambda \in \mathcal{P}, \lambda=1^{*} \text { or } \lambda=1^{* *} ; \\
-1, & \text { if } \lambda=1,\end{cases} \\
& c^{*}(\lambda):= \begin{cases}c(\lambda), & \text { if } \lambda \in \mathcal{P} ; \\
0, & \text { if } \lambda=1 ; \\
1, & \text { if } \lambda=1^{*} ; \\
-1, & \text { if } \lambda=1^{* *},\end{cases}
\end{aligned}
$$




$$
\sigma^{*}(\lambda):= \begin{cases}\sigma(\lambda), & \text { if } \lambda \in \mathcal{P} \\ 1, & \text { otherwise }\end{cases}
$$

Let

$$
\mathcal{W}_{2}=\left\{\left(\lambda_{1}, \lambda_{2}, \lambda_{3}, \lambda_{4}, \lambda_{5}, 2 \lambda_{6}, 2 \lambda_{7}\right) \mid \lambda_{1} \in \mathcal{D} \mathcal{E}, \lambda_{2}, \lambda_{3}, \lambda_{4}, \lambda_{5} \in \mathcal{D O}, \lambda_{6}, \lambda_{7} \in \mathcal{P}^{*}\right\}
$$

For a vector partition $\vec{\lambda} \in \mathcal{W}_{2}$, we define the sum of parts function $\widetilde{s}$, the weight function $\widetilde{w t}$ and the vector crank $c_{7}$ by

$$
\begin{aligned}
\widetilde{s}(\vec{\lambda}) & =\sigma\left(\lambda_{1}\right)+\sigma\left(\lambda_{2}\right)+\sigma\left(\lambda_{3}\right)+\sigma\left(\lambda_{4}\right)+\sigma\left(\lambda_{5}\right)+2 \sigma^{*}\left(\lambda_{6}\right)+2 \sigma^{*}\left(\lambda_{7}\right), \\
\widetilde{w t}(\vec{\lambda}) & =(-1)^{\ell\left(\lambda_{1}\right)} w t^{*}\left(\lambda_{6}\right) w t^{*}\left(\lambda_{7}\right), \\
c_{7}(\vec{\lambda}) & =\ell\left(\lambda_{2}\right)-\ell\left(\lambda_{3}\right)+2\left(\ell\left(\lambda_{4}\right)-\ell\left(\lambda_{5}\right)\right)+c^{*}\left(\lambda_{6}\right)+2 c^{*}\left(\lambda_{7}\right) .
\end{aligned}
$$

Let $M^{*}(m, n)$ denote the number of weighted 7 -colored partitions in $\mathcal{W}_{2}(n)$ with vector crank equal to $m$, and $M^{*}(k, m, n)$ denote the number of partitions in $\mathcal{W}_{2}(n)$ with vector crank congruent to $k$ modulo $m$. We have

$$
\sum_{m=-\infty}^{\infty} \sum_{n=0}^{\infty} M^{*}(m, n) z^{m} q^{n}=\frac{\left(q^{2} ; q^{2}\right)_{\infty}^{3}}{\left(z q, z^{-1} q, z^{2} q, z^{-2} q ; q\right)_{\infty}}
$$

Theorem 3.6. The following relation holds for all $n \geq 0$,

$$
M^{*}(0,7,7 n+4)=M^{*}(1,7,7 n+4)=\cdots=M^{*}(6,7,7 n+4)=\frac{w_{2}(7 n+4)}{7} .
$$

Proof. Setting $z=\zeta_{7}=e^{2 \pi i / 7}$ in (3.11), we have

$$
\begin{aligned}
& \sum_{m=-\infty}^{\infty} \sum_{n=0}^{\infty} M^{*}(m, n) \zeta_{7}^{m} q^{n} \\
& \quad=\frac{\left(q^{2} ; q^{2}\right)_{\infty}^{3}}{\left(\zeta_{7} q, \zeta_{7}^{-1} q, \zeta_{7}^{2} q, \zeta_{7}^{-2} q ; q\right)_{\infty}} \\
& =\frac{\left(q^{2} ; q^{2}\right)_{\infty}^{3}\left(\zeta_{7}^{3} q, \zeta_{7}^{-3} q, q ; q\right)}{\left(\zeta_{7} q, \zeta_{7}^{-1} q, \zeta_{7}^{2} q, \zeta_{7}^{-2} q, \zeta_{7}^{3} q, \zeta_{7}^{-3} q, q ; q\right)_{\infty}} \\
& =\frac{\sum_{m=0}^{\infty} \sum_{n=-\infty}^{\infty}(-1)^{m+n}(2 m+1) q^{m(m+1)+n(n+1) / 2}\left(1-\left(\zeta_{7}^{3}\right)^{2 n+1}\right)}{\left(q^{7} ; q^{7}\right)_{\infty}\left(1-\zeta_{7}^{3}\right) \zeta_{7}^{3 n}}
\end{aligned}
$$

Here the last equality follows from (2.22) and (3.9) again. The rest of proof is similar to our proof of Theorem 3.4. We therefore omit the details here.

\section{Partitions Weighted By the PARity of MUltirank AND VECTOR CRANK}

In [8], Choi, Kang, and Lovejoy studied arithmetic properties of the ordinary partition function weighted by the parity of the crank. Later, Kim [16] also studied arithmetic properties of cubic partition pairs weighted by the parity of the crank analog. As an analog 
of their work, we consider the number of weighted 7-colored partitions $w_{t}(n)$ weighted by the parity of the multirank and vector crank, i.e.,

$$
\begin{aligned}
\sum_{n=0}^{\infty} c_{t}(n) q^{n} & :=\sum_{n=0}^{\infty}\left(\sum_{m=-\infty}^{\infty}(-1)^{m} N_{\mathcal{V}_{t}}(m, n)\right) q^{n}=\frac{\left(q^{2} ; q^{2}\right)_{\infty}}{\left(q^{2} ; q^{4}\right)_{\infty}^{2}\left(q^{t} ; q^{t}\right)_{\infty}^{2}} \\
\sum_{n=0}^{\infty} d(n) q^{n} & :=\sum_{n=0}^{\infty}\left(\sum_{m=-\infty}^{\infty}(-1)^{m} M^{*}(m, n)\right) q^{n}=\left(q^{2} ; q^{2}\right)_{\infty}
\end{aligned}
$$

Interestingly, $c_{t}(n)$ satisfies the following equalities and congruences.

Theorem 4.1. For all $n \geq 0$,

$$
\begin{array}{lll}
c_{t}(5 n+3)=c_{t}(5 n+4)=0, & \text { if } t \equiv 0 \quad(\bmod 5), \\
c_{t}(5 n+4) \equiv 0 & (\bmod 5), & \text { if } t \equiv 1 \quad(\bmod 5), \\
c_{t}(5 n+3) \equiv 0 & (\bmod 5), & \text { if } t \equiv 4 \quad(\bmod 5) .
\end{array}
$$

Proof. Notice that, when $t \equiv 0(\bmod 5)$,

$$
\sum_{n=0}^{\infty} c_{t}(n) q^{n}=\frac{\left(q^{2} ; q^{2}\right)_{\infty}}{\left(q^{2} ; q^{4}\right)_{\infty}^{2}\left(q^{t} ; q^{t}\right)_{\infty}^{2}}=\frac{\left(q^{4} ; q^{4}\right)_{\infty}^{2}}{\left(q^{2} ; q^{2}\right)_{\infty}\left(q^{t} ; q^{t}\right)_{\infty}^{2}}=\frac{1}{\left(q^{t} ; q^{t}\right)_{\infty}^{2}} \sum_{n=0}^{\infty} q^{n(n+1)}
$$

where we use (2.2). Since $n(n+1)$ is not congruent to 3 and 4 modulo 5 , (4.1) follows immediately.

When $t \not \equiv 0(\bmod 5)$, we have, modulo 5 ,

$$
\sum_{n=0}^{\infty} c_{t}(n) q^{n}=\frac{\left(q^{2} ; q^{2}\right)_{\infty}}{\left(q^{2} ; q^{4}\right)_{\infty}^{2}\left(q^{t} ; q^{t}\right)_{\infty}^{2}} \equiv \frac{\left(q^{4} ; q^{4}\right)_{\infty}^{2}\left(q^{t} ; q^{t}\right)_{\infty}^{3}}{\left(q^{2} ; q^{2}\right)_{\infty}\left(q^{5 t} ; q^{5 t}\right)_{\infty}}
$$

It follows from (2.2) that

$$
\frac{\left(q^{2} ; q^{2}\right)_{\infty}^{2}}{(q ; q)_{\infty}}=P_{0}+P_{1}+P_{3}
$$

where $P_{i}$ consists of those terms in which the power of $q$ is $i$ modulo 5. Similar to the proofs of (2.19) and (2.20), one can easily obtain (4.2) and (4.3). This finishes the proof.

Remark 4.2. We first note that (4.1)-(4.3) are interesting as it appears to be very rare that the original partition function $w_{t}(n)$ and its weighted versions (which are weighted by the parity of its partition statistics) satisfy the same Ramanujan-type congruences. (Here (4.1) can be viewed as mod 5 congruences like (2.18).)

On the other hand, one readily sees that $c_{4}(n)=p\left(\frac{n}{2}\right)$ where $p(n)$ is the number of partitions of $n$ and $p(x)=0$ if $x$ is not an integer. From Ramanujan's congruences for $p(n)$ modulo powers of 5 , it is easy to get $c_{4}\left(5^{\alpha} n+\lambda_{\alpha}\right) \equiv 0\left(\bmod 5^{\alpha}\right)$, where $\lambda_{\alpha}$ is the least positive reciprocal of 12 modulo $5^{\alpha}$. Interestingly, this family of congruences resembles Sellers' result [19] for $c \phi_{2}(n)$ : 
Theorem 4.3 (Corollary 2.12, [18]). For all $n \geq 0$ and $\alpha \geq 1$,

$$
c \phi_{2}\left(5^{\alpha} n+\lambda_{\alpha}\right) \equiv 0 \quad\left(\bmod 5^{\alpha}\right),
$$

where $\lambda_{\alpha}$ is the least positive reciprocal of 12 modulo $5^{\alpha}$.

Relying on the combinatorial version of Euler's pentagonal number theorem [1, p. 10, Theorem 1.6], we obtain the following interesting corollary.

Corollary 4.4. Let $M_{e}^{*}(n)\left(\right.$ resp. $\left.M_{o}^{*}(n)\right)$ denote the number of weighted \%-colored partitions counted by $w_{2}(n)$ with even (resp. odd) vector crank. Then

$$
d(n)=M_{e}^{*}(n)-M_{o}^{*}(n)= \begin{cases}(-1)^{m}, & \text { if } n=m(3 m \pm 1) ; \\ 0, & \text { otherwise. }\end{cases}
$$

\section{FINAL REMARKS}

At last, we collect several questions here to motivate further investigation.

1) Yee [20] provided a combinatorial proof of the generating function for $k$-colored $\mathrm{F}$ partitions, which was independently established by Garvan [13]. To the best of our knowledge, there is no combinatorial proof for $k$-colored F-partition congruences up to now. In this paper, we provide a multirank that can explain the congruence modulo 5 combinatorially for $w_{4}(n)$. However, the combinatorial correspondence between $w_{4}(n)$ and $c \phi_{2}(n)$ is unclear. New ideas are required to emerge for solving these questions.

2) As shown in Sect. 3, we are able to combinatorially interpret mod 5 and 7 congruences for $w_{t}(n)$ by using a unified multirank or a vector crank. However, for mod 11 congruence (2.24), we cannot find such an interpretation along this line and hence we cry out for a combinatorial proof.

3) It is well known that Euler's pentagonal number theorem has a beautiful combinatorial proof, which was found by Franklin [11] in 1882. Hence it is also interesting to find a Franklin-type proof of the difference between $M_{e}^{*}(n)$ and $M_{o}^{*}(n)$ in (4.4).

\section{ACKNOWLEDGEMENT}

The authors would like to thank George E. Andrews, Shishuo Fu, Michael D. Hirschhorn and Ae Ja Yee for their helpful comments and suggestions that have improved this paper to a great extent. The authors also acknowledge the helpful suggestions made by the referee. The second author was supported by the National Natural Science Foundation of China (No. 11501061).

\section{REFERENCES}

[1] G. E. Andrews, The theory of partitions, Encyclopedia of Mathematics and its Applications, Vol. 2. Addison-Wesley Publishing Co., Reading, Mass.-London-Amsterdam, 1976. xiv +255 pp. (Reprinted: Cambridge University Press, London and New York, 1984). 6, 14

[2] G. E. Andrews, Generalized Frobenius partitions, Mem. Amer. Math. Soc. 49 (1984), no. 301, iv+44 pp. $1,2,4$ 
[3] G. E. Andrews and F. G. Garvan, Dyson's crank of a partition, Bull. Amer. Math. Soc. (N.S.) 18 (1988), no. 2, 167-171. 11

[4] N. D. Baruah and K. K. Ojah, Some congruences deducible from Ramanujan's cubic continued fraction, Int. J. Number Theory 7 (2011), no. 5, 1331-1343. 3

[5] A. Berkovich and F. G. Garvan, K. Saito's conjecture for nonnegative eta products and analogous results for other infinite products, J. Number Theory 128 (2008), no. 6, 1731-1748. 9

[6] B. C. Berndt, Ramanujan's notebooks. Part III, Springer-Verlag, New York, 1991. xiv +510 pp. 3

[7] B. C. Berndt, Number theory in the spirit of Ramanujan, Student Mathematical Library, 34. American Mathematical Society, Providence, RI, 2006. $\mathrm{xx}+187$ pp. 7

[8] D. Choi, S.-Y. Kang, and J. Lovejoy, Partitions weighted by the parity of the crank, J. Combin. Theory Ser. A 116 (2009), no. 5, 1034-1046. 12

[9] S. Cooper, M. D. Hirschhorn, and R. Lewis, Powers of Euler's product and related identities, Ramanujan J. 4 (2000), no. 2, 137-155. 8

[10] S. Fu and D. Tang, Multiranks and classical theta functions, Int. J. Number Theory, in press. 2

[11] F. Franklin, Sur le développement du produit infini $(1-x)\left(1-x^{2}\right)\left(1-x^{3}\right)\left(1-x^{4}\right) \ldots$, Comptes rendus 82 (1881), 448-450. 14

[12] F. G. Garvan, New combinatorial interpretations of Ramanujan's partition congruences mod 5, 7 and 11, Trans. Amer. Math. Soc. 305 (1988), no. 1, 47-77. 10

[13] F. G. Garvan, Generalizations of Dyson's rank, Thesis (Ph.D.), The Pennsylvania State University. 1986. 136 pp. 14

[14] B. Kim, An analog of crank for a certain kind of partition function arising from the cubic continued fraction, Acta Arith. 148 (2011), no. 1, 1-19. 11

[15] B. Kim, Partition statistics for cubic partition pairs, Electron. J. Combin. 18 (2011), no. 1, Paper 128,7 pp. 11

[16] B. Kim, Cubic partition pairs weighted by the parity of the crank, Korean J. Math. 23 (2015), no. 4, 637-642. 12

[17] J. Lovejoy, Ramanujan-type congruences for three colored Frobenius partitions, J. Number Theory 85 (2000), no. 2, 283-290. 2

[18] P. Paule and C.-S. Radu, The Andrews-Sellers family of partition congruences, Adv. Math. 230 (2012), no. 3, 819-838. 14

[19] J. Sellers, Congruences involving F-partition functions, Internat. J. Math. Math. Sci. 17 (1994), no. 1, 187-188. 13

[20] A. J. Yee, Combinatorial proofs of generating function identities for F-partitions, J. Combin. Theory Ser. A 102 (2003), no. 1, 217-228. 14

(Shane Chern) Department of Mathematics, The Pennsylvania State University, UniverSITY PARK, PA 16802, USA

E-mail address: shanechern@psu.edu; chenxiaohang92@gmail.com

(Dazhao Tang) College of Mathematics and Statistics, Chongqing University, Huxi CamPus LD206, Chongqing 401331, P.R. China

E-mail address: dazhaotang@sina.com 